\title{
25-hydroxyvitamin D status, light exposure and sleep quality in UK dwelling South Asian and Caucasian postmenopausal women
}

\section{A.L.Darling ${ }^{1,2}$, K.H.Hart ${ }^{1}$, S.Arber ${ }^{3}$, J.L.Berry ${ }^{4}$, P.L.Morgan ${ }^{2}$, B.A.Middleton ${ }^{2}$, S.Lanham-New ${ }^{1}$ and D.J.Skene ${ }^{2}$}

1 Department of Nutritional Sciences, School of Biosciences and Medicine, Faculty of Health and Medical Sciences, University of Surrey, Guildford, Surrey, GU2 7XH, UK

2 Chronobiology, Faculty of Health and Medical Sciences, University of Surrey, Guildford, Surrey, GU2 7XH, UK.

3 Department of Sociology, Faculty of Arts and Social Sciences, University of Surrey, Guildford, Surrey, GU2 7XH, UK

4 Specialist Assay Laboratory, Clinical Biochemistry, Manchester Royal Infirmary M13 9WL, UK

Short title: 25-hydroxyvitamin D status, light exposure and sleep quality

Authorship:

Designed protocol/conceived study: SA; DJS; KHH; SLN

Data collection: ALD; SLN

Data analysis: ALD; PLM; JLB; BAM; DJS; SLN

Wrote paper: ALD; DJS

Reviewed paper KHH; SA; JLB; PLM; BAM; DJS; SLN

Declarations of interests- DJS and BAM are co-directors of Stockgrand Ltd and Surrey Assays Ltd. SLN is Research Director of D3-TEX Ltd which holds the UK and Gulf Corporation Council (GCC) patent for the use of UVB transparent clothing to prevent vitamin D deficiency. All other authors have no interests to declare.

Correspondence: Professor Debra J. Skene; Chronobiology, Faculty of Health and Medical Sciences, University of Surrey, Guildford, Surrey, GU2 7XH, UK. Telephone: $+44(0) 1483$ 689706; Fax: +44(0)1483689712 email: d.skene@surrey.ac.uk 
This post-referee version is released with a Creative Commons Attribution Non-Commercial No Derivatives License. Please cite this article in press as: Darling AL et al. 25-hydroxyvitamin D status, light exposure and sleep quality in UK dwelling South Asian and Caucasian postmenopausal women J Steroid Biochem Mol Biol (2019) (in press).

Link to publisher final version:

https://www.sciencedirect.com/science/article/abs/pii/S0960076018303856?via\%3Dihub 


\section{Abstract}

There is a lack of research into 25-hydroxyvitamin $\mathrm{D}(25(\mathrm{OH}) \mathrm{D})$ status, light exposure and sleep patterns in South Asian populations. In addition, results of research studies are conflicting as to whether there is an association between $25(\mathrm{OH}) \mathrm{D}$ status and sleep quality.

We investigated $25(\mathrm{OH}) \mathrm{D}$ status, self-reported and actigraphic sleep quality in $\mathrm{n}=35$ UK dwelling postmenopausal women ( $n=13$ South Asians, $n=22$ Caucasians), who kept daily sleep diaries and wore wrist-worn actiwatch (AWL-L) devices for 14 days. A subset of $n=27$ women ( $n=11$ South Asian and $n=16$ Caucasian) also wore a neckworn AWL-L device to measure their light exposure.

For 25(OH)D concentration, South Asians had a median \pm IQR of $43.8 \pm 28.2 \mathrm{nmol} / \mathrm{L}$, which was significantly lower than Caucasians $(68.7 \pm 37.4 \mathrm{nmol} / \mathrm{L})(\mathrm{P}=0.001)$. Similarly, there was a higher sleep fragmentation in the South Asians (mean \pm SD 36.9 $\pm 8.9)$ compared with the Caucasians $(24.7 \pm 7.1)(P=0.002)$. Non-parametric circadian rhythm analysis of rest/activity patterns showed a higher night-time activity (L5) (22.6 \pm 14.0 vs. $10.5 \pm 4.4 ; P=0.0008)$ and lower relative amplitude $(0.85 \pm 0.07$ vs. $0.94 \pm 0.02 ; \mathrm{P}<0.0001)$ in the South Asian compared with the Caucasian women. More South Asians (50\%) met the criteria for sleep disorders (PSQI score $>5$ ) than did Caucasians (27\%) ( $\mathrm{P}=0.001$, Fishers Exact Test). However, there was no association between $25(\mathrm{OH}) \mathrm{D}$ concentration and any sleep parameter measured $(\mathrm{P}>0.05)$ in either ethnic group. South Asians spent significantly less time in illuminance levels over 200 lux ( $P=0.009)$ than did Caucasians.

Overall, our results show that postmenopausal South Asian women have lower 25(OH)D concentration than Caucasian women. They also have higher sleep fragmentation, as well as a lower light exposure across the day. This may have 
detrimental implications for their general health and further research into sleep quality and light exposure in the South Asian ethnic group is warranted.

Keywords Actigraphy, ethnicity, D-FINES study, daily light profile, illuminance thresholds 


\subsection{Introduction}

There is a lack of data on serum 25-hydroxyvitamin $D(25(\mathrm{OH}) \mathrm{D})$ in western dwelling South Asian ethnic groups but the work that has been done shows higher rates of vitamin $D$ deficiency $(<25 \mathrm{nmol} / \mathrm{L})$ and insufficiency $(<50 \mathrm{nmol} / \mathrm{L})$ compared with the native population of the country in which they reside [1-6]. The importance of 25(OH)D in ensuring bone and immune health, as well as possible prevention of chronic diseases such as cancer, cardiovascular disease and type 1 diabetes[7], means that it is crucial that we have an understanding of $25(\mathrm{OH}) \mathrm{D}$ concentrations in western dwelling South Asian groups.

It is known that sleep varies by ethnic group. Studies of rest-activity cycles and sleep quality have shown ethnic differences in key sleep characteristics [8, 9]. However, most studies assessing ethnicity and sleep quality have been conducted in the USA and have focussed on African American, White Caucasian, Hispanic, and Japanese populations. To date there are few studies investigating the prevalence of sleeprelated problems in western dwelling South Asian groups, with only one study having assessed European South Asians (Surinamese) living in the Netherlands [10]. This study found increased prevalence of self-reported short sleep in South Asian Surinamese compared with their native Dutch counterparts [10]. This study, however, only used self-reported measures, not objective actigraphic sleep measurements.

In addition, only a few studies have assessed sleep quality in South Asians residing in South Asia. Most of this research has been in men, with sleep problems attributed to sleep apnoea, exacerbated by obesity [11]. One recent study from India that did investigate both sexes found, in normal weight individuals, that $10.3 \%$ of South Asian 
women (compared with $8.3 \%$ of men) reported poor sleep, as assessed by the Pittsburgh Sleep Quality Index (PSQI) [12]. However, there has been no research to assess whether the incidence of sleep problems or actigraphic sleep differs between western dwelling South Asian women and their Caucasian counterparts.

Exposure to light is essential for sleep health as light is the primary zeitgeber (time cue) regulating the timing of circadian rhythms, and robust circadian cycles are required to maintain optimum sleep timing. There are no data to the authors' knowledge on light exposure in South Asian populations, but we have shown previously that UK South Asian women have lower ultraviolet B (UVB) exposure than Caucasian women [1], explaining their lower $25(\mathrm{OH}) \mathrm{D}$, but also suggesting that the total visible light exposure in this population might be lower than in Caucasian women. Theoretically, if South Asian women have lower visible light exposure then they may also have poorer sleep. This would be of public health concern due to the known detrimental effects of poor sleep and sleep deprivation on general health, including increased risk of chronic diseases such as cardiovascular disease, type 2 diabetes and obesity [13]. This is of particular concern in the South Asian population due to their already known increased risk of cardiovascular disease [14] and type 2 diabetes [15]. Moreover, an association has been found between short sleep duration in South Asian Surinamese and increased obesity risk [10].

Finally, it is unknown whether there is an association between vitamin D status and sleep quality in any ethnic group. Some studies have found that higher $25(\mathrm{OH}) \mathrm{D}$ concentrations are associated with reduced relative risk of insomnia[16] and lower daytime sleepiness[17]. The MrOS sleep study ( $n=3048$ older men) found shorter 
sleep duration and lower sleep efficiency in those with poorer $25(\mathrm{OH}) \mathrm{D}$ status after controlling for key confounders[18]. One study found that control subjects, matched for body mass index(BMI), had higher $25(\mathrm{OH}) \mathrm{D}$ concentrations than did mild to moderate obstructive sleep apnoea patients (OSA)[19]. However, more evidence is needed, especially in South Asians for which the relationship between 25(OH)D and sleep quality has not be assessed to date.

The aim of the present study was to assess $25(\mathrm{OH}) \mathrm{D}$ status, actigraphic rest-activity cycles, individual light exposure and subjectively reported sleep quality in UK dwelling South Asian and Caucasian women. Drawing on the existing evidence presented above, our hypotheses were as follows: South Asian women would have lower 25(OH)D status, lower light exposure, poorer self-reported sleep quality and poorer actigraphic sleep, than age- and latitude-matched Caucasian women. It was also predicted that lower $25(\mathrm{OH}) \mathrm{D}$ status would be associated with poorer sleep quality in both ethnic groups. 


\subsection{Materials and Methods}

\subsection{Participants}

Study participants were recruited from within a convenience sample of postmenopausal women taking part in the D-FINES (Vitamin D, Food Intake, Nutrition and Exposure to Sunlight in Southern England) II study at the University of Surrey in 2010. D-FINES II was a cross sectional study of South-East UK dwelling women of South Asian and European Caucasian origin. Ethnicity was self-reported by the participants. These participants had originally been recruited from a database of women who had attended for vitamin D research studies at the University of Surrey (D-FINES I), details of the recruitment and methods for which have been published elsewhere [1]. Briefly, participants were recruited via General Practitioner (GP) databases in a variety of geographical areas of differing socio-economic status in the South-East of England, with $n=365$ both premenopausal and postmenopausal women taking part in the initial study.

Participants were invited back to the University of Surrey for further study measurements (D-FINES II) in Summer 2010, with $n=82$ postmenopausal women taking part. These women were also invited to take part in the present 14-day light and sleep study, with a total of $n=35$ women agreeing to participate $[n=13$ South Asian $62.3 \pm 2.7 y($ mean $\pm S D)$ and $n=22$ Caucasian women 66.4 $\pm 4.9 y]$. Figure 1 illustrates the flow of participants from the D-FINES II cohort into the present study.

The subgroup who took part in the light and sleep study $(n=35$, Supplementary Table 1) were similar in age, alcohol intake and smoking status to those women who did not take part ( $n=47$, Supplementary Table 2). $P$ values could not be obtained for analysis 
of variables for the South Asian women who did take part vs. those who did not, due to a very small number of South Asian women not taking part ( $n=4$ to 8 , depending on variable). However, the South Asian women who participated were on average obese $\left(\mathrm{BMl} \geq 30 \mathrm{~kg} / \mathrm{m}^{2}, \mathrm{n}=13\right)$ compared with those who not did take part, who were on average overweight (BMl of $26-20 \mathrm{~kg} / \mathrm{m}^{2}, \mathrm{n}=4$ ). In addition, those who took part in the study came from households with a slightly lower deprivation index (i.e. lower index of multiple deprivation score, less deprived neighbourhood), although this did not reach statistical significance (Caucasians, $P=0.17$ ).

In accordance with the ethical standards (1964 Declaration of Helsinki), ethical approvals were obtained from the relevant Research Ethics Committees (National Health Service 10/H1109/25) and the University of Surrey Research Ethics Committee (EC/2010/53/FHMS). Written, informed consent was obtained from all participants.

\subsection{Assessment of 25(OH)D concentration}

The manual enzyme immunoassay (Immunodiagnostic Systems Ltd, Boldon, Tyne and Wear, UK) was used to measure $25(\mathrm{OH}) \mathrm{D}$ concentration. Reference ranges given by the manufacturer are $48-144 \mathrm{nmol} / \mathrm{L}$ and vary by season. Manufacturer's values for intra- and inter-assay coefficients of variation were $6 \%$ and $7 \%$, respectively and sensitivity was $5 \mathrm{nmol} / \mathrm{L}(2 \mathrm{ng} / \mathrm{ml})[1]$. Measurement of 25(OH)D was undertaken by the Vitamin D Research Group based at the Specialist Assay laboratory Manchester Royal Infirmary, UK. The laboratory participated successfully in the Vitamin D quality assurance scheme (DEQAS) and was accredited to standards ISO 9001:2008 and ISO 13485:2003. 


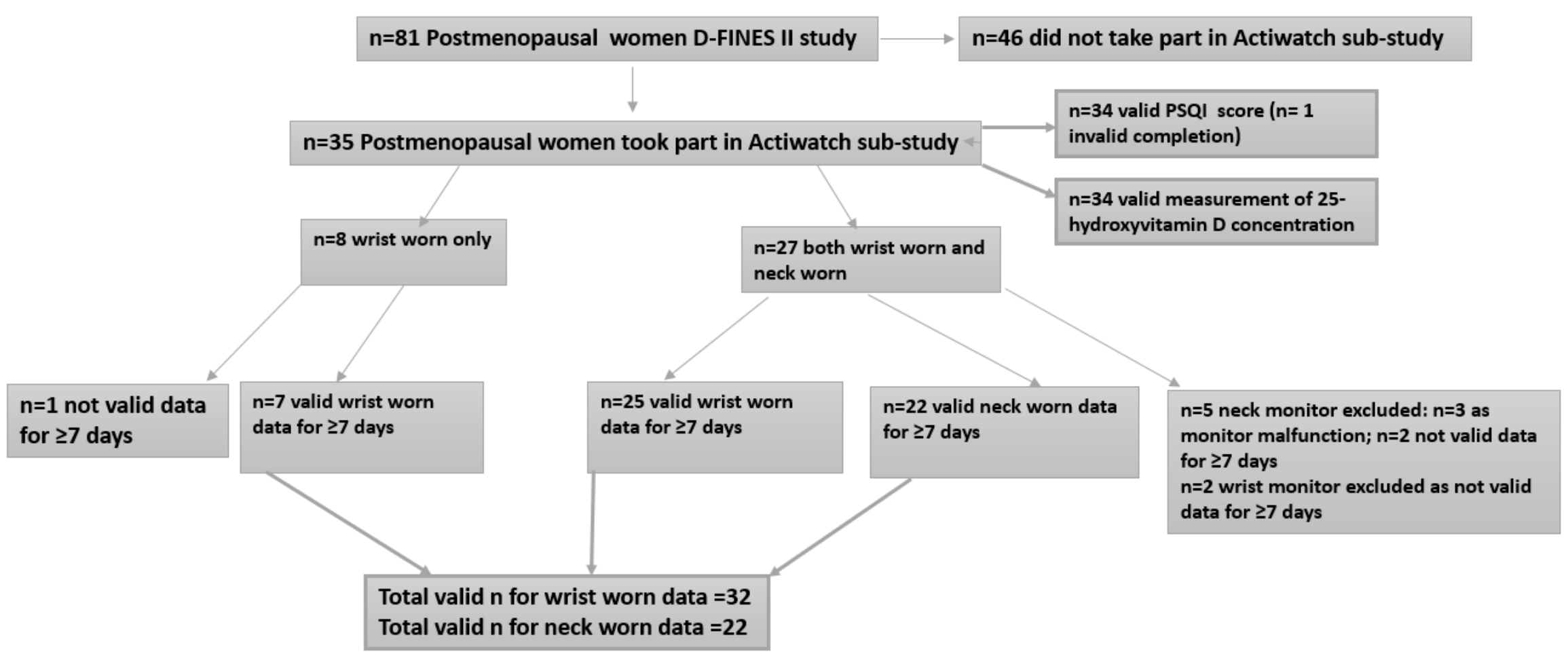

2 Figure 1. Flow chart of subject participation in the light and sleep (Actiwatch) sub-study, including number of participants 3 included in the 25-hydroxyvitamin D, sleep and light analyses 


\section{$5 \quad 2.3$ Actigraphy and sleep diaries}

6 Participants ( $n=35 ; n=13$ South Asian and $n=22$ Caucasian) wore an Actiwatch-L

7 (AWL-L; Cambridge Neurotechnology Ltd, Cambridge, UK) on their non-dominant

8 wrist for assessment of rest-activity cycles as a proxy for sleep/wake patterns and

9 sleep quality, for 14 consecutive days, 24 hours a day, recording in one minute epochs.

10 Fourteen days was deemed the minimum duration for this study [20]. A subset of participants also wore an AWL-L on a neck cord $(n=27 ; n=11$ South Asian and $n=16$ Caucasian) during waking hours, over the same 14 days as they wore the wrist AWL$\mathrm{L}$, in order to more accurately assess ocular light exposure. See Figure 1 for details of the number of participants who wore wrist worn AWL-L only, or who wore both wrist and neck worn AWL-L.

All participants were asked to keep a record of when the watch and/or neck monitors were worn and removed and were asked to ensure clothing did not cover the light sensors. They also completed a sleep diary each morning, to record sleep timing, sleep interruptions and to rate their perceived sleep quality, details of which have been published previously [21]. All participants completed the Munich Chronotype Questionnaire (MCTQ) to assess their chronotype (namely, mid-sleep on free days, corrected for sleep debt on work days(MSFsc)) and degree of social jetlag (SJL) [22]. between $20^{\text {th }}$ September $2010-25^{\text {th }}$ October 2010 , with sunrise between $05: 45$ to 06:42 $\mathrm{h}$ and sunset between 16:50 to $18: 08 \mathrm{~h}$. The study was scheduled so that all participants would finish the study before the clocks changed from British Summer 
30 Ramadan period, when rest-activity cycles are likely to change in the South Asian women, the majority of whom were Muslims.

Participants started the study in different weeks due to insufficient number of AWL-L units to start all participants at the same time. To minimise the effect of variation in light levels and weather during the different study weeks, wearing of the AWL-L devices was balanced as far as possible between the two study groups (see Supplementary File for further details).

\subsection{Light data analysis}

The data obtained from both the wrist and neck worn AWL-L were analysed using the standard software provided by the manufacturer (Sleep Analysis 5, Cambridge Neurotechnology Ltd). For the neck worn AWL-L, the amount of time spent above three reference illuminance thresholds (200, 500 and 1000 lux) was calculated for each participant over the 14 days. Any days where compliance with wearing the neck AWL-L was problematic (i.e. no movement for $>3$ hours/day) were discarded. Any participant with $<7$ days remaining had all their data removed from the analysis, as at least 5-6 days of data are advised for adequate reliability in adults [23].

Daily 24 hour time profiles were produced for each participant who had worn an AWL$\mathrm{L}$ around their neck, showing the mean of the median illuminance for each hour of the day over the 14 days. Prior to analysis, daytime zero values (likely to be accidental coverage of the AWL-L with clothing) were removed using a macro (created by Peter

53 Williams, University of Surrey) in SAS software (SAS Institute, Cary, NC, USA), to reduce data skewness. 


\subsection{Actigraphic sleep and circadian rhythm analysis}

57 The earliest time to bed and latest wake time, as recorded in the 14 day sleep diaries, were used to inform the analysis of each participant's actigraphic sleep. Any period between 10 minutes and 3 hours where the AWL-L appeared not to have been worn was replaced with the daily mean. Non-parametric circadian rhythm analysis (NPCRA) was also performed on the data from each participant's wrist worn AWL-L, over the 14 days, using a Microsoft Excel Macro (provided by E.J. Van Someren). The NPCRA analysis enabled estimation of 8 rest-activity parameters; the 5 hours of lowest activity (L5), the 10 hours of highest activity (M10), time of onset of L5 (L5 onset), time of onset of M10 (M10 onset), amplitude, relative amplitude, inter-daily stability (IS) and intra-daily variability (IV) [24]. PSQI scores were calculated using the standard scoring system [25].

\subsection{Statistical analysis}

Ethnic differences in 25(OH)D were assessed using the Mann-Whitney test, due to skewness in the $25(\mathrm{OH}) \mathrm{D}$ measurements which could not be normalised using a log transform. Ethnic differences in light exposure were assessed using independent $t$ tests. The AWL-L and self-reported sleep parameters were normally distributed according to the Kolmogorov-Smirnov test, except for sleep latency, which was normalised by log transformation. Independent t-tests were used to assess ethnic differences in the actigraphic parameters of sleep efficiency, sleep fragmentation, log sleep latency as well as the subjectively reported parameters of 'time try to sleep', 'sleep duration' and 'wake time'. 
NPCRA parameters were not normally distributed and log transformation failed to normalise IS and relative amplitude, so these were analysed using Mann-Whitney's U test. Independent t-tests were used to assess the other log transformed NPCRA parameters. PSQI scores are ordinal data so ethnic differences were assessed using the Chi-square test for percentages of participants in categories and the MannWhitney $U$ test for global PSQI scores and subscale differences. The association between 25(OH)D and sleep variables were assessed using Pearson's or Spearman's Rho correlations as appropriate.

87 In terms of multiple testing, 25- $(\mathrm{OH}) \mathrm{D}$ concentrations, light data, objective and subjective sleep data and associations between light and sleep parameters were viewed as 4 groups (each referring to a separate hypothesis), Bonferroni adjustment for multiple testing being applied within groups. All tests were undertaken using SPSS version 21 (Chicago, IL).

\subsection{Results}

93

\subsection{Demographic data for ethnic groups}

Participant characteristics for subjects with complete wrist worn $(n=32)$ or neck worn 96 $(\mathrm{n}=22)$ AWL-L data for $\geq 7$ days are shown in Table 1. 
Table 1: Participant Characteristics- all subjects with complete AWL-L (wrist worn or neck worn) data for $\geq 7$ days

\begin{tabular}{|c|c|c|c|c|c|c|c|c|c|c|c|c|c|c|}
\hline \multirow[b]{3}{*}{ Parameter } & \multicolumn{7}{|c|}{ Complete wrist worn AWL data for $\geq 7$ days $n=32$} & \multicolumn{7}{|c|}{ Complete neck worn AWL data for $\geq 7$ days $n=22$} \\
\hline & \multicolumn{3}{|c|}{ CAUCASIAN } & \multicolumn{3}{|c|}{ SOUTH ASIAN } & \multirow[t]{2}{*}{$\mathbf{P}^{¥}$} & \multicolumn{2}{|c|}{ CAUCASIAN } & \multirow[b]{2}{*}{ SD } & \multicolumn{3}{|c|}{ SOUTH ASIAN } & \multirow[b]{2}{*}{$\mathbf{P}^{¥}$} \\
\hline & $\mathbf{N}$ & Mean & SD & $\mathbf{n}$ & Mean & SD & & $\mathbf{n}$ & Mean & & $\mathbf{n}$ & Mean & SD & \\
\hline Age (years) & 22 & 66.4 & 4.9 & 10 & 62.6 & 2.3 & 0.006 & 16 & 66.3 & 4.6 & 6 & 63.2 & 2.1 & 0.124 \\
\hline IMD & 21 & 13.8 & 7.7 & 10 & 13.1 & 12.6 & 0.845 & 16 & 13.9 & 8.8 & 6 & 11.2 & 11.8 & 0.568 \\
\hline BMI $\left(\mathrm{kg} / \mathrm{m}^{2}\right)$ & 22 & 24.9 & 4.9 & 10 & 32.2 & 4.3 & $<0.001$ & 16 & 24.9 & 5.4 & 6 & 33.8 & 4.2 & 0.002 \\
\hline Caffeine- cups of tea/d & 22 & 2.7 & 2.1 & 10 & 3.0 & 1.2 & 0.730 & 16 & 3.0 & 2.3 & 6 & 3.1 & 1.0 & 0.909 \\
\hline Caffeine- cups of coffee/d & 22 & 1.3 & 2.3 & 9 & 0.1 & 0.3 & 0.022 & 16 & 1.3 & 2.6 & 6 & 0.2 & 0.5 & 0.392 \\
\hline Mid-sleep (MSFsc) & 19 & 3.4 & 0.5 & 9 & 3.9 & 1.1 & 0.256 & 14 & 3.5 & 0.5 & 6 & 3.4 & 0.9 & 0.061 \\
\hline Social Jetlag (SJL) h & 19 & 0.2 & 0.3 & 9 & 0.5 & 0.7 & 0.286 & 14 & 0.2 & 0.3 & 6 & 0.2 & 0.3 & 0.772 \\
\hline \multirow[t]{2}{*}{ Number of days in work/ week $\neq$} & 19 & 1.0 & 7.0 & 9 & 0.0 & 7.0 & 0.920 & 14 & 0.5 & 7.0 & 6 & 0.0 & 7.0 & 0.718 \\
\hline & $\mathbf{N}$ & $\% \mathrm{Y}$ & $\% \mathrm{~N}$ & $\mathbf{n}$ & $\% \mathrm{Y}$ & $\% \mathrm{~N}$ & $\begin{array}{l}\text { Fisher } \\
\text { Exact }\end{array}$ & $\mathbf{n}$ & $\% \mathrm{Y}$ & $\% \mathrm{~N}$ & $\mathbf{n}$ & $\% \mathrm{Y}$ & $\% \mathbf{N}$ & $\begin{array}{l}\text { Fisher } \\
\text { Exact }\end{array}$ \\
\hline Sleep medication user & 22 & 0 & 100 & 8 & 25 & 75 & 0.064 & 16 & 0 & 100 & 4 & 0 & 100 & $\neq$ \\
\hline Smoker & 22 & 9 & $91^{ \pm}$ & 10 & 0 & 100 & $>0.999$ & $16 \pm$ & 0 & 100 & 6 & 0 & 100 & $\neq$ \\
\hline 0-4 units alcohol/wk & \multicolumn{3}{|c|}{$54 \%(\mathrm{n}=12)$} & \multicolumn{3}{|c|}{$100 \%(\mathrm{n}=9)$} & 0.030 & \multicolumn{3}{|c|}{$63 \%((\mathrm{n}=10)$} & \multicolumn{3}{|c|}{$100 \%(\mathrm{n}=5)$} & 0.262 \\
\hline $\begin{array}{l}\text { 5+ units alcohol/wk } \\
\text { Mid-sleep on free days (MSFsc) }\end{array}$ & \multicolumn{3}{|c|}{$46 \%(\mathrm{n}=10)$} & \multicolumn{4}{|c|}{$0 \% \quad(\mathrm{n}=0)$} & \multicolumn{3}{|c|}{$37 \%(\mathrm{n}=6)$} & \multicolumn{3}{|c|}{$0 \%(\mathrm{n}=0)$} & \\
\hline$\leq 3.0$ & \multicolumn{3}{|c|}{$26.3 \%(\mathrm{n}=5)$} & \multicolumn{3}{|c|}{$22.2 \%(n=2)$} & $>0.999$ & \multicolumn{3}{|c|}{$28.6 \%(n=4)$} & \multicolumn{3}{|c|}{$33.3 \%(\mathrm{n}=2)$} & $>0.999$ \\
\hline$>3.0$ & \multicolumn{3}{|c|}{$73.7 \%(n=14)$} & \multicolumn{3}{|c|}{$77.8 \%(\mathrm{n}=7)$} & & \multicolumn{3}{|c|}{$71.4 \%(\mathrm{n}=12)$} & \multicolumn{3}{|c|}{$66.6 \%(\mathrm{n}=4)$} & \\
\hline $\begin{array}{l}\text { Social Jetlag (SJL) h } \\
\leq 0.50\end{array}$ & \multicolumn{3}{|c|}{$84.2 \%(n=16)$} & \multicolumn{3}{|c|}{$66.7 \%(\mathrm{n}=6)$} & 0.352 & \multicolumn{3}{|c|}{$78.6 \%(\mathrm{n}=11)$} & \multicolumn{3}{|c|}{$83.3 \%(\mathrm{n}=5)$} & $>0.999$ \\
\hline$>0.50$ & \multicolumn{3}{|c|}{$15.8 \%(n=3)$} & 33.3 & $(n=3)$ & & & 21.4 & $=3)$ & & & $=1)$ & & \\
\hline
\end{tabular}


After Bonferroni correction, independent t-tests showed a statistically significant difference in $\mathrm{BMI}(\mathrm{P}<0.001)$ with the South Asian group having a higher $\mathrm{BMI}$ compared with the Caucasian group (32 vs. $25 \mathrm{~kg} / \mathrm{m}^{2}$ ) (Table 1). According to the English Index of Multiple Deprivation (IMD)(see Supplementary File for details of this metric) there was not a statistically significant ethnic difference in socio-economic status (IMD=13.8 in Caucasian group vs. 13.1 in South Asian group, $\mathrm{P}=0.85$ ). South Asian women worked a median (interquartile range (IQR)) of $0(7)$ days per week, compared with Caucasian who worked a median (IQR) of 1 (7) days per week, (ns, P=0.92). Demographic characteristics for participants with complete neck worn AWL-L data for $\geq 7$ days $(n=22)$, are also presented in Table 1 , with characteristics similar to those who wore the wrist-worn AWL-L only $(n=32)$. Equivalent data for all $n=35$ participants (including those who were excluded from analyses $(n=3)$ due to having $<7$ days of valid AWL-L data) can be found in Supplementary Table 1.

\subsection{Ethnic differences in 25-hydroxyvitamin D status}

For $n=35$ women, (including the $n=3$ women excluded from the Actiwatch analyses due to AWL-L compliance issues), $n=34$ had $25(\mathrm{OH}) \mathrm{D}$ concentration data. South Asian women $(n=12)$ had significantly lower $25(\mathrm{OH})$ D concentrations (median (IQR) $43.8(28.2) \mathrm{nmol} / \mathrm{L})$ compared with the Caucasian group $(\mathrm{n}=22)(68.7(37.4) \mathrm{nmol} / \mathrm{L}$, Mann-Whitney $U$ test $P=0.001)$. Moreover, $58.3 \%$ of the South Asians had $25(\mathrm{OH}) \mathrm{D}$ $<50 \mathrm{nmol} / \mathrm{L}$, and $91.7 \%$ had $25(\mathrm{OH}) \mathrm{D}<75 \mathrm{nmol} / \mathrm{L}$. Equivalent figures for the Caucasian group were $4.5 \%<50 \mathrm{nmol} / \mathrm{L}$ and $59.1 \%<75 \mathrm{nmol} / \mathrm{L}$. No participants had $25(\mathrm{OH}) \mathrm{D}<25 \mathrm{nmol} / \mathrm{L}$. Approximately half of the South Asians (54\%) and the Caucasians (45\%) reported having taken a vitamin D supplement in the past 3 years (i.e. since the end of the D-FINES I study), the majority in both groups being combined 
calcium and vitamin D supplements prescribed by their General Practitioner (Family Physician).

\subsection{Ethnic differences in objective and subjective sleep parameters}

Independent samples t-tests showed that South Asians had a lower sleep efficiency

131

132 (mean (SD): 80(7) vs. 88(4); $\mathrm{P}=0.006$ ) and higher sleep fragmentation (mean (SD): 37(9) vs. 25(7); $P=0.002$ ) (Figure 2). However, there was no significant difference in sleep latency by ethnic group $(\mathrm{P}=0.76)$. For the NPCRA parameters, independent samples t-tests showed a significantly lower amplitude in South Asians $(253 \pm 84$ vs. 323 $\pm 83 ; P=0.037)$ but no ethnic difference in IV $(P=0.32)$. Mann Whitney $U$ test showed a significantly lower relative amplitude in the South Asians $(0.85 \pm 0.07$ vs. $0.94 \pm 0.02 ; P<0.0001)$ but no ethnic difference in IS $(P=0.10)$. (Table 2$)$. Independent samples t-tests showed that South Asians had a significantly higher L5 (22.6 $6 \pm 14.0$ vs. $10.5 \pm 4.4 ; P=0.0008)$ but there was no ethnic difference in M10, L5 onset time or M10 onset time.

In terms of self-reported sleep from the daily sleep diaries, independent t-tests showed no statistically significant ethnic differences in sleep duration, time 'try to sleep' or wake time (Table 2). Fisher Exact Tests showed that a higher percentage of the South Asian group (50\%) met the criteria for sleep disorders (PSQI score $>5$ ) compared to the Caucasian group (27\%) $(P=0.001)$. There was a statistically significant ethnic difference in global PSQI score (median (IQR)= 6.0 (8.5) in South Asians vs. 3.5 (4.25) in Caucasians; $\mathrm{P}=0.04)$ and in the sleep duration (DURAT) subscale (median $(I Q R)=$ $1.5(3)$ in South Asians vs. $0(1)$ in Caucasians, $P=0.006)$. 
However, the only findings which survived Bonferroni adjustment for multiple testing

152 (23 tests, $\mathrm{P} \leq 0.002$ ) were the higher percentage of South Asian women meeting the

153 criteria for sleep disorders, as well as the lower relative amplitude, higher L5 (night-

154 time activity), and higher sleep fragmentation in South Asian women. Actigraphic sleep

155 efficiency had a borderline statistically significant association $(P=0.006)$ with the South

156 Asian group having reduced sleep efficiency.

157

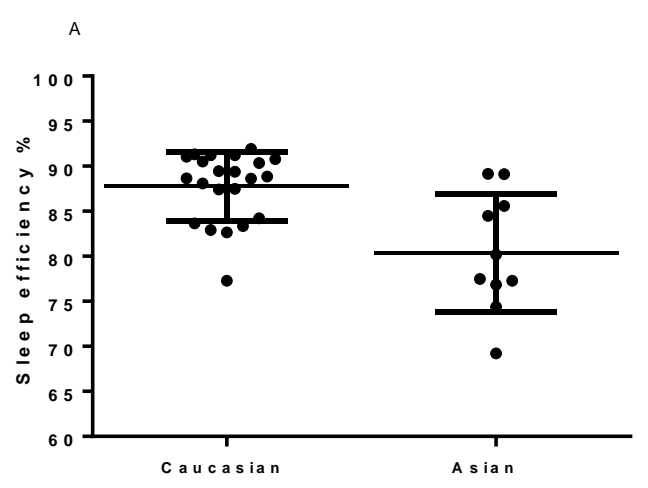

$n=22$

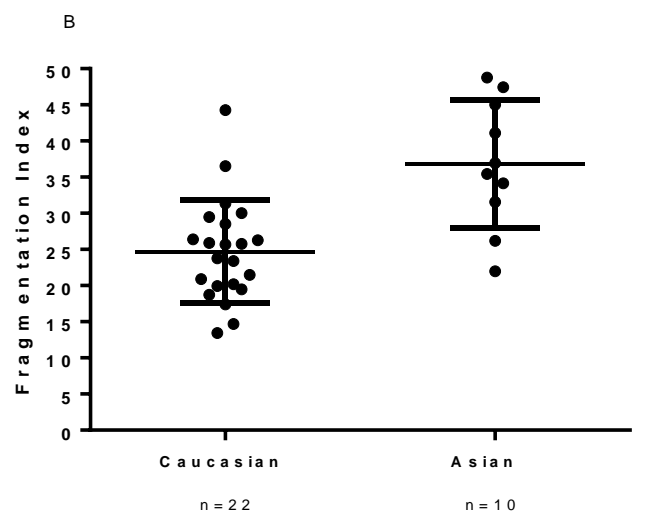

158

Figure 2: Ethnic differences in actigraphic parameters: A. Sleep efficiency and $(\mathbf{n}=10)$ women. Individual and mean (SD) data derived from wrist-worn AWL-L, averaged over $\geq 7$ days. South Asian women had significantly reduced sleep efficiency $(P=0.006)$ and increased sleep fragmentation $(P=0.002)$ compared to the Caucasian women. 


\begin{tabular}{|c|c|c|c|c|c|c|c|}
\hline \multirow[b]{2}{*}{ Parameter } & \multirow[b]{2}{*}{$\mathbf{n}$} & \multicolumn{2}{|c|}{ CAUCASIAN } & \multicolumn{2}{|c|}{ SOUTH ASIAN } & \multicolumn{2}{|r|}{$\mathbf{P}^{¥}$} \\
\hline & & Mean & SD & $\mathbf{n}$ & Mean & SD & Independent t-test \\
\hline \multicolumn{8}{|l|}{ Light Exposure } \\
\hline Dec.h over 200 lux & 16 & 4.24 & 1.11 & 6 & 2.86 & 0.56 & 0.009 \\
\hline Dec.h over 500 lux & 16 & 2.45 & 0.75 & 6 & 1.53 & 0.48 & 0.012 \\
\hline \multirow[t]{2}{*}{ Dec.h over 1000 lux } & 16 & 1.47 & 0.52 & 6 & 0.98 & 0.39 & 0.048 \\
\hline & $\mathbf{n}$ & Median & IQR & & Median & IQR & Mann-Whitney \\
\hline AUC for $24 h$ Time Profile & 16 & 4624 & 2848 & 6 & 2700 & 2090 & 0.08 \\
\hline \multirow[t]{2}{*}{ AUC $\leq \mathbf{1 1 . 3 0 ~ h ~}$} & 16 & 860 & 501 & 6 & 323 & 180 & 0.01 \\
\hline & $\mathbf{n}$ & Mean & SD & $\mathbf{n}$ & Mean & SD & $\begin{array}{c}\text { Independent t-test/ } \\
\text { Mann Whitney } ¥\end{array}$ \\
\hline \multicolumn{8}{|l|}{ AWL-L } \\
\hline Sleep Latency* (mins) & 22 & 14 & 11 & 10 & 20 & 20 & 0.759 \\
\hline Sleep Efficiency (\%) & 22 & 87.74 & 3.81 & 10 & 80.37 & 6.57 & 0.006 \\
\hline Sleep Fragmentation & 22 & 24.71 & 7.09 & 10 & 36.86 & 8.88 & 0.002 \\
\hline \multicolumn{8}{|l|}{ NPCRA } \\
\hline Amplitude & 22 & 323 & 83 & 10 & 253 & 84 & 0.037 \\
\hline Relative Amplitude $¥$ & 22 & 0.94 & 0.02 & 10 & 0.85 & 0.07 & $<0.001$ \\
\hline IS $¥$ & 22 & 0.59 & 0.1 & 10 & 0.52 & 0.07 & 0.101 \\
\hline IV & 22 & 0.79 & 0.1 & 10 & 0.85 & 0.2 & 0.322 \\
\hline L5 & 22 & 10.5 & 4.4 & 10 & 22.6 & 14.0 & 0.0008 \\
\hline M10 & 22 & 333 & 85 & 10 & 275 & 92 & 0.092 \\
\hline L5 onset (h:min) & 22 & $1: 00$ & $1: 05$ & 10 & 1:00 & $1: 12$ & 0.921 \\
\hline M10 onset (h:min) & 22 & $8: 42$ & $0: 42$ & 10 & 9:06 & $1: 30$ & 0.309 \\
\hline
\end{tabular}




\begin{tabular}{|c|c|c|c|c|c|c|c|}
\hline Subjective- sleep diary & & & & & & & \\
\hline Sleep duration (Dec. $h$ ) & 22 & 8.01 & 0.68 & 10 & 7.77 & 1.10 & 0.539 \\
\hline Time 'try to sleep' (mins) & 22 & 23.37 & 0.62 & 10 & 23.57 & 0.62 & 0.402 \\
\hline Wake time (Dec. h) & 22 & 7.38 & 0.59 & 10 & 7.34 & 0.97 & 0.908 \\
\hline PSQI & $\mathbf{n}$ & Median & IQR & $\mathbf{n}$ & Median & IQR & Mann Whitney \\
\hline Global score- & 22 & 3.5 & 4.25 & 12 & 6.0 & 8.5 & 0.040 \\
\hline Sleep Duration & 22 & 0 & 1 & 12 & 1.5 & 3 & 0.006 \\
\hline Sleep disturbances & 22 & 1 & 1 & 12 & 1.5 & 1 & 0.110 \\
\hline Sleep latency & 22 & 1 & 1 & 12 & 1 & 2 & 0.327 \\
\hline Daytime dysfunction & 22 & 0 & 1 & 12 & 0 & 2 & 0.736 \\
\hline Habitual Sleep Efficiency & 22 & 0 & 1 & 12 & 0.5 & 3 & 0.683 \\
\hline Sleep Quality & 22 & 1 & 1 & 12 & 1 & 2 & 0.231 \\
\hline Sleep Medication & 22 & 0 & 0 & 12 & 0 & 2 & 0.118 \\
\hline
\end{tabular}

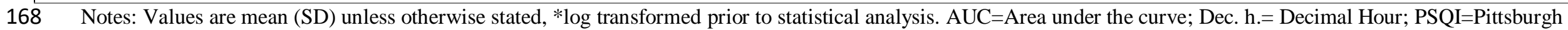

169 Sleep Quality Index. ¥ Mann-Whitney test rather than Independent t-test 


\subsection{Ethnic differences in light exposure}

171 Daily light profiles across the $24 \mathrm{~h}$ day for the two ethnic groups are shown in Figure

3. The main period of light exposure for both groups occurred between 07:30 $\mathrm{h}$ and 19:30 h. Peak light intensity occurred at 13:30 h in South Asians and at 16:30 h in Caucasians. In terms of overall intensity of light received, there was a non-statistically significant trend $(P=0.08)$ for the South Asian group (median (IQR) of $2700 \pm 2090$ units) to have a lower Illuminance throughout the day than the Caucasian group (median (IQR) of $4624 \pm 2848$ units) (Table 2 ), indicating reduced total daily light exposure by $58 \%$ in the South Asians. Of note, when looking at morning light exposure up to and including $11.30 \mathrm{~h}$, there was significantly reduced exposure (38\%) in the South Asians compared to that of Caucasians (323 \pm 180 units vs. $860 \pm 501$ units; $\mathrm{P}=0.01$, Table 2). Independent t-tests showed that South Asians spent less time in lighting over $200(P=0.009), 500(P=0.012)$ and 1000 lux $(P=0.048)$ than Caucasians (Table 2, Figure 4). However only the ethnic difference in time spent over 200 lux $(P=0.009)$ and ethnic difference in light exposure up to and including $11.30 \mathrm{~h}(\mathrm{P}=0.01)$ were statistically significant following Bonferroni adjustment (5 tests, revised cut-off $P \leq 0.01)$. 
A

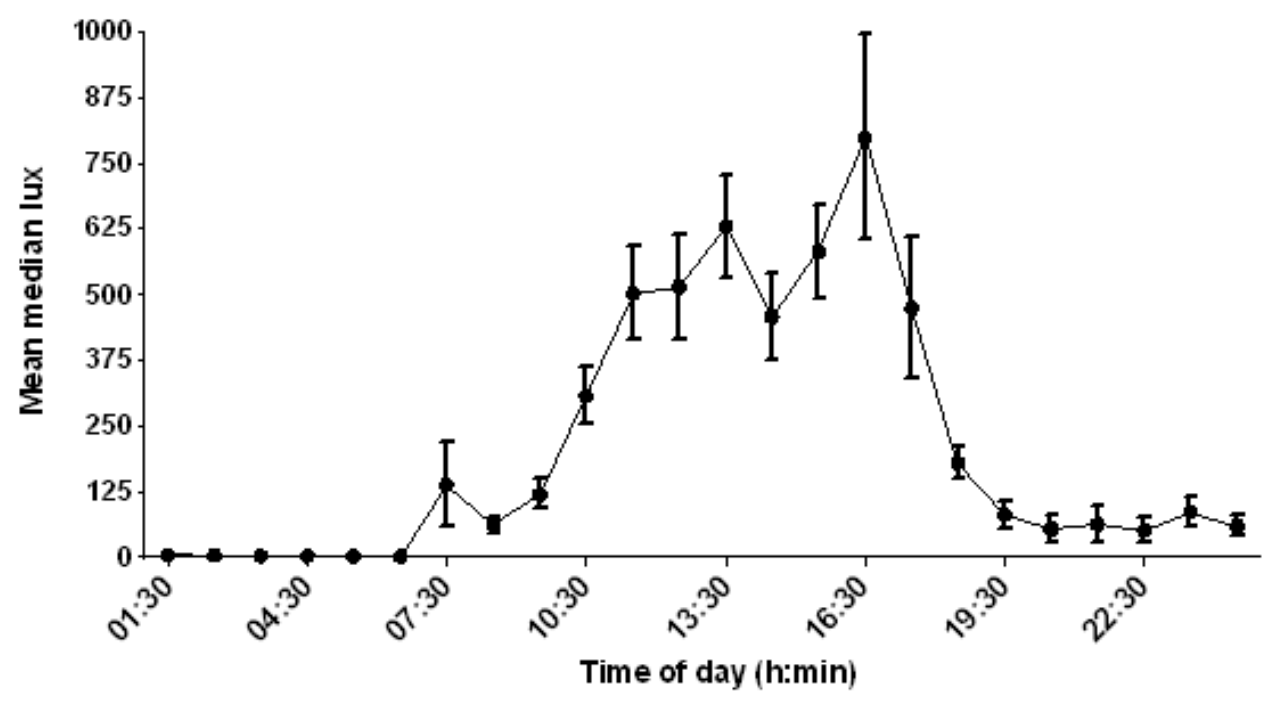

B

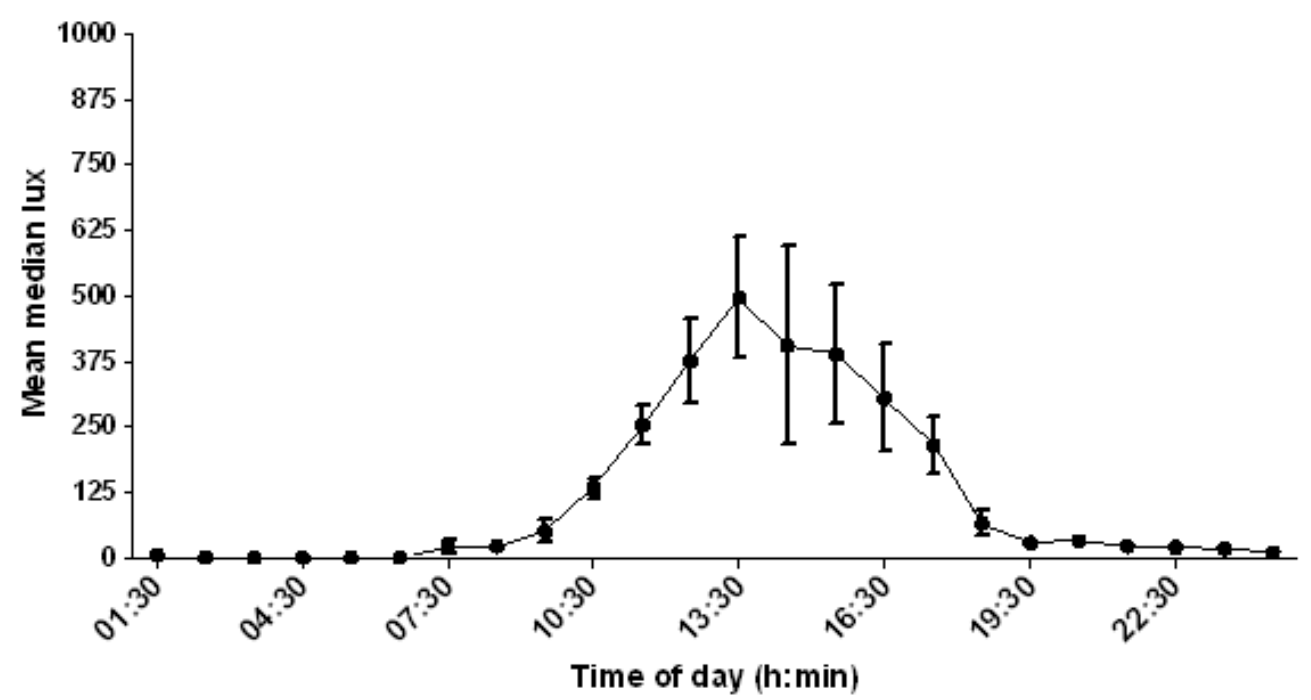

Figure 3. Daily light profiles across $24 \mathrm{~h}$ for $A$. Caucasian $(n=16)$ and B. South Asian ( $\mathbf{n}=6)$ women. Hourly mean median illuminance readings (lux)( $\pm S E M)$ were derived from neck worn AWL-L, averaged over $\geq 7$ days. 


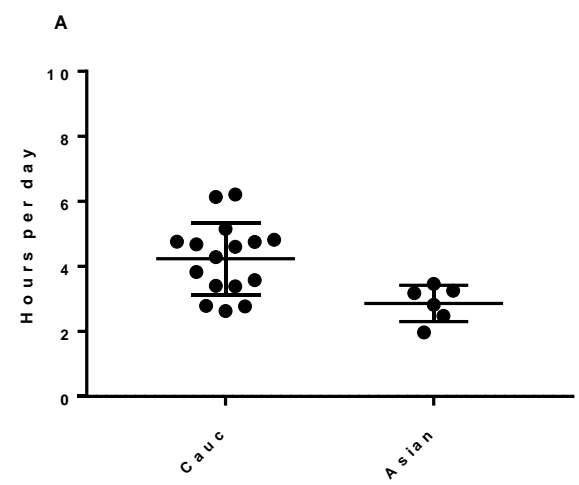

Independent test

B

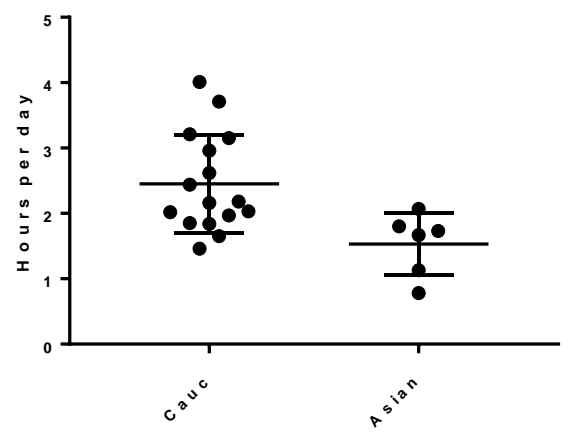

Independent t-tes

$p=0.012$

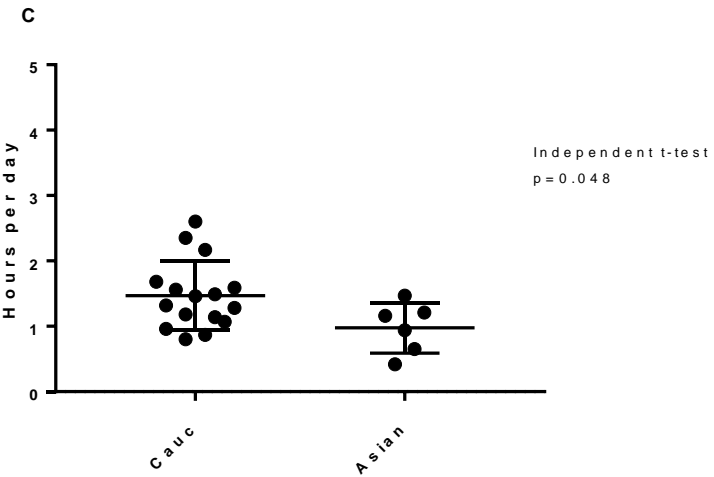

198 Figure 4. Hours per day spent over different light thresholds. Hours per day (Dec.h) spent 199 in illuminance levels A. > 200 lux; B. > 500 lux and C. > 1000 lux. Individual values and 200 mean \pm SD are plotted for Caucasians $(n=16)$ and South Asians $(n=6)$. Cauc=Caucasian, 201 Asian= South Asian. 


\subsection{Association between vitamin D status and sleep parameters}

205 Pearson's correlations were run between 25(OH)D concentrations and the self206 reported time 'try to sleep', sleep duration and wake time $(n=31)$ (Table 3), with no 207 significant associations identified in either ethnic group ( $P>0.05)$. Spearman's Rho correlations were also run between $25(\mathrm{OH}) \mathrm{D}$ and global and sub-scale scores for the PSQI questionnaire $(n=31)$ and there were no significant associations between 25(OH)D and any of these PSQI variables in either ethnic group (Table 3).

212 Partial correlations were also run between 25(OH)D and AWL-L sleep parameters 213 (Pearson's), as well as NPCRA-derived parameters (Spearman's Rho) (n=31) (Table $2143)$. There were no significant associations between any of these parameters and $21525(\mathrm{OH}) \mathrm{D}$ in either ethnic group $(\mathrm{P}>0.05)$. 

and NPCRA sleep parameters and $25(\mathrm{OH})$ D- all study participants $(n=31)^{\neq}$

\begin{tabular}{|c|c|c|c|c|c|c|}
\hline & \multicolumn{3}{|c|}{ Caucasians } & \multicolumn{3}{c|}{ Asians } \\
\hline $\begin{array}{c}\text { Subjective sleep parameters } \\
\text { (Spearman's Rho) }\end{array}$ & $\mathbf{r}$ & $\mathbf{p}$ & $\mathbf{n}$ & $\mathbf{r}$ & $\mathbf{p}$ & $\mathbf{N}$ \\
\hline Time 'try to sleep' (Dec.h) & 0.168 & 0.455 & 22 & 0.033 & 0.932 & 9 \\
\hline Subjectively reported sleep duration & -0.021 & 0.926 & 22 & -0.167 & 0.667 & 9 \\
\hline Self-reported wake time (Dec.h) & 0.096 & 0.672 & 22 & 0.001 & $>0.99$ & 9 \\
\hline Global PSQI score & -0.005 & 0.982 & 22 & -0.067 & 0.864 & 9 \\
\hline Sleep duration & 0.087 & 0.699 & 22 & 0.137 & 0.724 & 9 \\
\hline Sleep disturbances & -0.144 & 0.523 & 22 & -0.529 & 0.143 & 9 \\
\hline Sleep latency & 0.248 & 0.266 & 22 & -0.163 & 0.675 & 9 \\
\hline Daytime dysfunction & 0.220 & 0.325 & 22 & 0.269 & 0.484 & 9 \\
\hline Habitual Sleep Efficiency & 0.042 & 0.851 & 22 & -0.419 & 0.262 & 9 \\
\hline Sleep quality & -0.104 & 0.646 & 22 & -0.077 & 0.856 & 9 \\
\hline Sleep medication & $¥$ & $¥$ & 22 & -0.139 & 0.720 & 9 \\
\hline AWL sleep parameters (Pearson's) & & & & & & \\
\hline Sleep efficiency (\%) & 0.09 & 0.691 & 22 & -0.276 & 0.473 & 9 \\
\hline Sleep fragmentation (\%) & 0.004 & 0.985 & 22 & 0.491 & 0.179 & 9 \\
\hline Sleep latency (Dec.h) & 0.126 & 0.576 & 22 & -0.273 & 0.477 & 9 \\
\hline NPCRAt: (Spearman's Rho) & & & & & & \\
\hline IS & -0.158 & 0.484 & 22 & 0.244 & 0.527 & 9 \\
\hline IV & -0.217 & 0.333 & 22 & 0.183 & 0.637 & 9 \\
\hline L5 & -0.037 & 0.871 & 22 & 0.217 & 0.576 & 9 \\
\hline L5 onset (Dec.h) & 0.249 & 0.264 & 22 & -0.400 & 0.286 & 9 \\
\hline M10 & 0.242 & 0.278 & 22 & 0.217 & 0.576 & 9 \\
\hline M10 onset (Dec.h) & 0.149 & 0.509 & 22 & -0.317 & 0.406 & 9 \\
\hline Amplitude & 0.225 & 0.315 & 22 & 0.133 & 0.732 & 9 \\
\hline & 0.148 & 0.510 & 22 & -0.184 & 0.635 & 9 \\
\hline
\end{tabular}
daily stability, IV = intra-daily variability, L5 $=5$ hours with lowest activity, L5onset $=$ time of onset of 5 hours of lowest activity, M10 = 10 hours with most activity, M10 onset = time of onset of 10 hours of most activity, Amplitude = (M10 L5), Relative amplitude $=$ amplitude/(M10+L10). $¥$ no data as score for MEDS=0 for all Caucasians on this scale; 


\subsection{Discussion}

We found significantly lower 25(OH)D status in postmenopausal South Asian women compared with age-matched Caucasian women. On average the South Asian women had 25(OH)D concentrations of $44 \mathrm{nmol} / \mathrm{L}$, compared with $69 \mathrm{nmol} / \mathrm{L}$ in the Caucasian women. This finding concurs with previous research on western dwelling South Asian men and women, who have been found to have lower $25(\mathrm{OH}) \mathrm{D}$ levels than the native population of the country in which they reside [1-6]. In the current study, $58 \%$ of the South Asian women had $25(\mathrm{OH}) \mathrm{D}$ lower than $50 \mathrm{nmol} / \mathrm{L}$, the current recommendation for health by the US Institute of Medicine for Health[26]. Public health action is thus urgently required to remedy this vitamin $D$ insufficiency, although only one woman in the study had severe vitamin $D$ deficiency $(<25 \mathrm{nmol} / \mathrm{L})$.

Some of the ethnic differences in $25(\mathrm{OH}) \mathrm{D}$ concentrations seen here may be due to increased adiposity in the South Asian group, but it is also likely due to lifestyle differences, including that of sun avoidance [27], as well as low intake of vitamin D from food [28]. Many women in our South Asian group were taking vitamin D supplements, and thus it is likely that the true $25(\mathrm{OH}) \mathrm{D}$ status of the UK wide South Asian population is a lot lower, as most UK dwelling South Asians do not take vitamin D containing supplements[28].

In terms of sleep quality, we found significantly higher sleep fragmentation and nighttime activity (L5) and a lower relative rest-activity amplitude in South Asian women, compared with Caucasian women. The South Asian women also self-reported significantly more sleep problems on the PSQI. Together these findings indicate poorer subjective and objective sleep, and a less robust rest-activity rhythm in the 
South Asian women. There are no previous studies using actigraphy in South Asian populations to compare our results with but our results concur with previous research

251

252

253

254

255

256

257

258

259

260

261

262

263

264

265

266

267

268

findings of poorer sleep in other western dwelling ethnic minority groups, compared with White Caucasian groups [29-31].

Our observed ethnic difference in sleep quality could be due to lifestyle and environmental differences. For example, ethnic minority groups are over represented in urban areas [32] which have increased night-time street light and increased ambient noise levels, with associated sleep disturbance [33]. However, both our ethnic groups came from housing areas with a similar deprivation index, suggesting this socioeconomic status may not be an explanatory factor for the ethnic differences seen. Nonetheless there may be other characteristics of South Asian households that are not measured by the deprivation index which could lead to sleep disruption and reduced light exposure (e.g. number of persons living in the household; adult children or elderly relatives living in home). The observed ethnic differences could also be due to differences in adiposity, as the South Asian participants were on average obese compared with the Caucasians who were on average overweight. Obesity has been suggested to adversely affect sleep quality [34]. Although we cannot rule out obesity as an explanation of the ethnic differences seen in this paper, equally it is not advisable to control for it in our analyses as it may lie on the causal pathway for the association between ethnicity and sleep quality.

Genetic differences in sleep and circadian timing related to ethnicity may also be important in explaining our results. Ethnic differences in circadian period length have been reported [35] as well as worldwide variation in the frequency of some clock 
genes, PER3 [36, 37] and CLOCK [37] gene variants. As we did not observe ethnic differences in sleep timing or chronotype our findings may reflect differences in sleep homeostasis rather than circadian timing.

In terms of subjectively reported sleep quality, PSQI scores indicated that South Asian women had a higher prevalence of sleep disorder (PSQI>5) than Caucasian women. The poorer sleep quality reported by the South Asians supports our objective actigraphic data of significantly reduced sleep efficiency and higher sleep fragmentation. The observed differences in sleep quality, however, could not be attributed simply to shorter sleep duration, or different sleep and wake times due to not finding statistically significant differences in these variables by ethnicity. Moreover, published data in the area of subjective sleep quality and South Asian ethnicity are sparse. Panda et al. using the PSQI found poor sleep (PSQI>5) in 10.3\% of India waking up too early in the morning [38]. The only published study in western dwelling South Asians reporting subjective sleep data was that of Anujuo et al. ([10], which found that female South Asian Surinamese had a higher prevalence of self-reported short sleep (<7h per night) compared with the native Dutch population. We did not find an ethnic difference in self-reported sleep duration in our study but this may be explained by study differences such as the older age of the women in our study as compared with that of Anujuo et al. [10].

It is unclear as to whether our observed ethnic differences in sleep are explainable by modifiable factors associated with South Asian ethnicity (e.g. increased adiposity, 
lower physical activity, increased use of sleep medications) or whether it is something fundamental to South Asian ethnicity itself (e.g. genetics). Future studies in South Asians are warranted to investigate this further using larger samples of different sexes and ages from a variety of socioeconomic backgrounds.

Our findings show that South Asian women spent significantly less time in light levels above 200 lux than Caucasian women. Daily $24 \mathrm{~h}$ light profiles also showed a trend for the South Asians to have lower morning light exposure (up to $11.30 \mathrm{~h}$ ) than the Caucasians, and a trend for less light exposure over the whole day. Ethnic differences in light exposure may be due to different cultural practices, for example, indoor activities and sun avoidance are encouraged in South Asians [27]. Although we did not directly assess this with our data due to the relatively small sample size, we can speculate that the lower light levels measured in the South Asians may be associated with the lower rest-activity rhythm amplitude observed in this group. A less robust restactivity circadian rhythm may adversely affect sleep maintenance, and overall lower light exposure could also directly affect sleep homeostasis. Less light exposure and reduced circadian amplitude may explain the poorer sleep quality seen in the South Asian participants, and this needs to be investigated with a larger sample size. However, it should be borne in mind that other factors such as obesity, and specifically obstructive sleep apnoea (OSA), may also explain poorer sleep quality in the South Asian group.

There is no previous literature with which to compare our light exposure data, as to date no studies have measured light exposure in western dwelling South Asians. Moreover, few studies have assessed ethnic differences in light exposure in any minority ethnic group. Kripke et al. found lower light exposure in Black and Hispanic 
women compared with European Caucasian women [31], however Jean-Louis et al. showed no difference in light exposure between European Caucasian and ethnic minority groups (Hispanics, Blacks)[29]. Our finding of lower light exposure and a less robust rest-activity rhythm amplitude in South Asian women supports these studies which suggested minority groups may show increased circadian dysregulation, with adverse consequences for sleep quality, in comparison with Caucasians.

Finally, we found no association between $25(\mathrm{OH}) \mathrm{D}$ status and sleep quality, neither for subjectively reported or actigraphically assessed sleep, in either ethnic group. This contradicts other previous work [16-18]. However our work with continuous recording across 14 days is of a smaller sample size, which could have contributed to the finding of no association, compared to these other studies. Also, only one of our research participants (a South Asian) had 25(OH)D less than 25nmol/L (deficiency), which was unexpected, particularly in South Asians whereby $25(\mathrm{OH}) \mathrm{D}<25 \mathrm{nmol} / \mathrm{L}$ is very common[3]. This is likely due to the high prevalence of vitamin D supplementation in the South Asian group as a result of having taken part in the D-FINES I study in 20062007 whereby many were diagnosed as vitamin D deficient and encouraged to take a vitamin D supplement by their general practitioner (family physician). The findings suggest that $25(\mathrm{OH}) \mathrm{D}$ needs to be quite low before there are any detrimental effects on sleep, although the other studies cited above that did find an association between $25(\mathrm{OH}) \mathrm{D}$ and sleep had similar $25(\mathrm{OH}) \mathrm{D}$ concentrations to our women [16-18]. Finally, the study by McCarty et al. [17] consisted of sleep clinic patients, who may differ from our non-clinical sample. 
There is no known reason, other than musculoskeletal pain caused by severe vitamin

D deficiency, why vitamin D may affect sleep. There is the assumption of a possible mechanism via the ubiquitous presence of the vitamin $D$ receptor and the vitamin $D$ hydroxylase enzymes throughout the human brain[39], meaning it is present in brain areas such as the hypothalamus [39] which in part controls sleep homeostasis. Conversely, a recent review paper postulated that the link between 25(OH)D status and sleep is mediated by pain, as well as inflammatory markers such as tumor necrosis factor alpha, prostaglandin D2 and interleukin-1 and obstruction or inflammation of the airway [40]. However, evidence for this explanation is currently limited and more research is required.

To the authors' knowledge, this work is the first to investigate $25(\mathrm{OH}) \mathrm{D}$ status, light exposure, subjective and objective sleep patterns and quality in a western dwelling South Asian population. Strengths of this work include the fact that actigraphy is a validated method for assessing rest-activity cycles and light exposure, and the PSQI is a validated method for assessing self-reported sleep quality. The subjects were also studied for 14 days in their natural setting, capturing a more realistic profile of sleep wake cycles than would be measured in the laboratory.

Limitations of this study include the fact that actigraphy has known limitations in assessing sleep characteristics since it uses arm movement as a proxy for wakefulness. In addition, the sub-study had a small sample size, especially for the South Asian group. This was exacerbated by the fact that some women had to be excluded from the analysis due to lack of compliance with wearing the AWL-L devices. 
372 In addition, Bonferroni correction, known to be highly conservative, may have resulted

373 in some type II errors, particularly those which initially had low $P$ values (e.g. $P<0.001)$.

374 There is a risk of selection bias due to the fact that not all D-FINES participants took

375 part in this sleep and light study. This may affect the generalisability of the results and

376 further research is required with a larger and more representative sample. Also, as

377 the D-FINES study originally was a study of vitamin D status and bone health, research participants were not screened for OSA, and a higher prevalence of OSA in the South Asian group could partly explain the results seen in this study.

It is not clear to what degree ethnic differences in self-reporting sleep reflect cultural norms for sleep quality, and how much is due to actual physiological differences in sleep. However, the self-reported PSQI results were in agreement with the objective actigraphic rest-activity parameters (e.g. sleep fragmentation) in the South Asian women, strengthening our findings. The research participants were also vitamin D sufficient on average $(\geq 50 \mathrm{nmol} / \mathrm{L})$ and associations between sleep quality and 25(OH)D need to be assessed further in vitamin $D$ deficient populations. Future studies in South Asians are warranted to investigate this further using larger samples of different sexes and ages from a variety of socioeconomic backgrounds.

\subsection{Conclusion}

Our study suggests the potential for sleep-related health problems in postmenopausal South Asian women. South Asian women had significantly lower 25(OH)D concentrations, poorer actigraphic sleep (higher sleep fragmentation, more night-time activity, a reduced relative rest-activity amplitude) as well as worse self-reported sleep 
396 than their Caucasian counterparts. However, no association between 25(OH)D 397 concentrations and sleep quality was observed. The South Asian group also had 398 significantly reduced light exposure (less time spent in light levels over 200 lux, and 399 less morning light exposure) than the Caucasian women.

400

401

402

Acknowledgements

403 Funding for this work was provided by the University of Surrey (PhD studentship for 404 ALD, D-FINES II study). We thank Dr Thomas Kantermann for help with the MCTQ 405 analysis. The authors would like to acknowledge those who helped with participant recruitment: Mrs Shahnaz Bano, Ms Judy Dudman, Mrs Roxanna Hanjra, Mrs Razia Killedar, Mrs Rohini Mahendran, Mrs Rafeea Mahoon, Mr John Pheasant and Mrs Freda Smithers. The original D-FINES I study (2006-2007) was funded by the UK Food Standards Agency (FSA, grant number N05064).

410

411

Dedication

412 This paper is dedicated to Mr John Pheasant, Practice Manager at Thornton Heath

413 Medical Centre, London who helped with the study recruitment for the D-FINES I 414 study and who sadly died in 2008. 


\section{References}

[1] A.L. Darling, K.H. Hart, H.M. Macdonald, K. Horton, A.R. Kang'ombe, J.L. Berry, S.A. Lanham-New, Vitamin D deficiency in UK South Asian Women of childbearing age: a comparative longitudinal investigation with UK Caucasian women, Osteoporos Int. 24 (2013) 477-488.

[2] H.M. Macdonald, A. Mavroeidi, W.D. Fraser, A.L. Darling, A.J. Black, L. Aucott, F. O'Neill, K. Hart, J.L. Berry, S.A. Lanham-New, D.M. Reid, Sunlight and dietary contributions to the seasonal vitamin D status of cohorts of healthy postmenopausal women living at northerly latitudes: a major cause for concern?, Osteoporos Int. 22 (2011) 2461-2472.

[3] N.M. Lowe, S.R. Mitra, P.C. Foster, I. Bhojani, J.F. McCann, Vitamin D status and markers of bone turnover in Caucasian and South Asian postmenopausal women living in the $\mathrm{UK}, \mathrm{Br}$ J Nutr. 103 (2010) 1706-1710.

[4] R. Kift, J.L. Berry, A. Vail, M.T. Durkin, L.E. Rhodes, A.R. Webb, Lifestyle factors including less cutaneous sun exposure contribute to starkly lower vitamin D levels in U.K. South Asians compared with the white population, Br J Dermatol. 169 (2013) 1272-1278.

[5] C. Hamson, L. Goh, P. Sheldon, A. Samanta, Comparative study of bone mineral density, calcium, and vitamin D status in the Gujarati and white populations of Leicester, Postgrad Med J. 79 (2003) 279-283.

[6] H.E. Meyer, J.A. Falch, A.J. Sogaard, E. Haug, Vitamin D deficiency and secondary hyperparathyroidism and the association with bone mineral density in persons with Pakistani and Norwegian background living in Oslo, Norway, The Oslo Health Study, Bone. 35 (2004) 412-417.

[7] M.F. Holick, Vitamin D deficiency, N Engl J Med. 357 (2007) 266-281.

[8] C.J. Stepnowsky, Jr., P.J. Moore, J.E. Dimsdale, Effect of ethnicity on sleep: complexities for epidemiologic research, Sleep. 26 (2003) 329-332.

[9] S.D. Sanford, K.L. Lichstein, H.H. Durrence, B.W. Riedel, D.J. Taylor, A.J. Bush, The influence of age, gender, ethnicity, and insomnia on Epworth sleepiness scores: a normative US population, Sleep Med. 7 (2006) 319-326.

[10] K. Anujuo, K. Stronks, M.B. Snijder, G. Jean-Louis, G. Ogedegbe, C. Agyemang, Ethnic differences in self-reported sleep duration in The Netherlands--the HELIUS study, Sleep Med. 15 (2014) 1115-1121.

[11] S. Agrawal, S.K. Sharma, V. Sreenivas, R. Lakshmy, Prevalence of metabolic syndrome in a north Indian hospital-based population with obstructive sleep apnoea, Indian J Med Res. 134 (2011) 639-644.

[12] S. Panda, A.B. Taly, S. Sinha, G. Gururaj, N. Girish, D. Nagaraja, Sleep-related disorders among a healthy population in South India, Neurol India. 60 (2012) 68-74.

[13] A. Shankar, S. Syamala, S. Kalidindi, Insufficient rest or sleep and its relation to cardiovascular disease, diabetes and obesity in a national, multiethnic sample, PloS one. 5 (2010) e14189.

[14] M.R. Boon, L.E. Bakker, R.A. van der Linden, A.F. van Ouwerkerk, P.L. de Goeje, J. Counotte, I.M. Jazet, P.C. Rensen, High prevalence of cardiovascular disease in South Asians: Central role for brown adipose tissue?, Crit Rev Clin Lab Sci. 52 (2015) 150-157.

[15] N. Sattar, J.M. Gill, Type 2 diabetes in migrant south Asians: mechanisms, mitigation, and management, Lancet Diabetes Endocrinol. 3 (2015) 1004-1016.

[16] M.A. Beydoun, A.A. Gamaldo, J.A. Canas, H.A. Beydoun, M.T. Shah, J.M. McNeely, A.B. Zonderman, Serum nutritional biomarkers and their associations with sleep among US adults in recent national surveys, PloS one. 9 (2014) e103490.

[17] D.E. McCarty, A. Reddy, Q. Keigley, P.Y. Kim, A.A. Marino, Vitamin D, race, and excessive daytime sleepiness, Journal of clinical sleep medicine : JCSM : official publication of the American Academy of Sleep Medicine. 8 (2012) 693-697.

[18] J. Massa, K.L. Stone, E.K. Wei, S.L. Harrison, E. Barrett-Connor, N.E. Lane, M. Paudel, S. Redline, S. Ancoli-Israel, E. Orwoll, E. Schernhammer, Vitamin D and actigraphic sleep outcomes in older community-dwelling men: the MrOS sleep study, Sleep. 38 (2015) 251-257. 
[19] C.P. Kerley, K. Hutchinson, K. Bolger, A. McGowan, J. Faul, L. Cormican, Serum Vitamin D Is Significantly Inversely Associated with Disease Severity in Caucasian Adults with Obstructive Sleep Apnea Syndrome, Sleep. 39 (2016) 293-300.

[20] E.J. Van Someren, Improving actigraphic sleep estimates in insomnia and dementia: how many nights?, J Sleep Res. 16 (2007) 269-275.

[21] V.S. Staples, S.N. Archer, S. Arber, D.J. Skene, Daily light exposure profiles in older nonresident extreme morning and evening types, J Sleep Res. 18 (2009) 466-471.

[22] T. Roenneberg, T. Kuehnle, M. Juda, T. Kantermann, K. Allebrandt, M. Gordijn, M. Merrow, Epidemiology of the human circadian clock, Sleep Med Rev. 11 (2007) 429-438.

[23] K.L. Knutson, P.J. Rathouz, L.L. Yan, K. Liu, D.S. Lauderdale, Intra-individual daily and yearly variability in actigraphically recorded sleep measures: the CARDIA study, Sleep. 30 (2007) 793-796.

[24] E.J. Van Someren, D.F. Swaab, C.C. Colenda, W. Cohen, W.V. McCall, P.B. Rosenquist, Bright light therapy: improved sensitivity to its effects on rest-activity rhythms in Alzheimer patients by application of nonparametric methods, Chronobiol Int. 16 (1999) 505-518.

[25] D.J. Buysse, C.F. Reynolds, 3rd, T.H. Monk, S.R. Berman, D.J. Kupfer, The Pittsburgh Sleep Quality Index: a new instrument for psychiatric practice and research, Psychiatry Res. 28 (1989) 193-213.

[26] IOM, Dietary reference intakes for calcium and vitamin D, The National Academies Press, Washington DC, 2011.

[27] P.R. von Hurst, W. Stonehouse, J. Coad, Vitamin D status and attitudes towards sun exposure in South Asian women living in Auckland, New Zealand, Public health nutrition. 13 (2010) 531-536.

[28] A.L. Darling, D.J. Blackbourn, K.R. Ahmadi, S.A. Lanham-New, Vitamin D supplement use and associated demographic, dietary and lifestyle factors in 8024 South Asians aged 4069 years: analysis of the UK Biobank cohort, Public health nutrition. (2018) 1-11.

[29] G. Jean-Louis, D.F. Kripke, S. Ancoli-Israel, M.R. Klauber, R.S. Sepulveda, Sleep duration, illumination, and activity patterns in a population sample: effects of gender and ethnicity, Biol Psychiatry. 47 (2000) 921-927.

[30] G. Jean-Louis, C.M. Magai, C.I. Cohen, F. Zizi, H. von Gizycki, J. DiPalma, G.J. Casimir, Ethnic differences in self-reported sleep problems in older adults, Sleep. 24 (2001) 926-933.

[31] D.F. Kripke, G. Jean-Louis, J.A. Elliott, M.R. Klauber, K.M. Rex, A. Tuunainen, R.D. Langer, Ethnicity, sleep, mood, and illumination in postmenopausal women, BMC Psychiatry. 4 (2004) 8.

[32] S. Garner, G. Bhattacharyya, Poverty, Ethnicity and Place. JRF programme paper: Poverty and Ethnicity [online], Joseph Rowntree Foundation, 2011.

[33] B. Jakovljevic, G. Belojevic, K. Paunovic, V. Stojanov, Road traffic noise and sleep disturbances in an urban population: cross-sectional study, Croat Med J. 47 (2006) 125-133. [34] T.A. Hargens, A.S. Kaleth, E.S. Edwards, K.L. Butner, Association between sleep disorders, obesity, and exercise: a review, Nat Sci Sleep. 5 (2013) 27-35.

[35] C.I. Eastman, T.A. Molina, M.E. Dziepak, M.R. Smith, Blacks (African Americans) have shorter free-running circadian periods than whites (Caucasian Americans), Chronobiol Int. 29 (2012) 1072-1077.

[36] A.A. Barbosa, M. Pedrazzoli, B.D. Koike, S. Tufik, Do Caucasian and Asian clocks tick differently?, Braz J Med Biol Res. 43 (2010) 96-99.

[37] C.M. Ciarleglio, K.K. Ryckman, S.V. Servick, A. Hida, S. Robbins, N. Wells, J. Hicks, S.A. Larson, J.P. Wiedermann, K. Carver, N. Hamilton, K.K. Kidd, J.R. Kidd, J.R. Smith, J. Friedlaender, D.G. McMahon, S.M. Williams, M.L. Summar, C.H. Johnson, Genetic differences in human circadian clock genes among worldwide populations, J Biol Rhythms. 23 (2008) 330-340.

[38] S. Stranges, W. Tigbe, F.X. Gomez-Olive, M. Thorogood, N.B. Kandala, Sleep problems: an emerging global epidemic? Findings from the INDEPTH WHO-SAGE study among more than 40,000 older adults from 8 countries across Africa and Asia, Sleep. 35 (2012) 1173-1181. 
[39] D.W. Eyles, S. Smith, R. Kinobe, M. Hewison, J.J. McGrath, Distribution of the vitamin D receptor and 1 alpha-hydroxylase in human brain, J Chem Neuroanat. 29 (2005) 21-30.

[40] D.E. McCarty, A.L. Chesson, Jr., S.K. Jain, A.A. Marino, The link between vitamin D metabolism and sleep medicine, Sleep Med Rev. 18 (2014) 311-319. 